\title{
AC Loss Measurements of Model and Full Size 50mm SSC Collider Dipole Magnets at Fermilab
}

J.P. Ozelis, S.W. Delchamps, S. Gourlay, T. Jaffery, W. Kinney, W. Koska, M. Kuchnir, M.J. Lamm, P.O. Mazur, D. Orris, J. Strait, M. Wake

Fermi National Accelerator Laboratory

P.O. Box 500, Batavia, Illinois 60510

J. Dimarco, J. Kuzminski and H. Zheng

SSC Laboratory

Dallas, Texas 75237

September 1992

Presented at the 1992 Applied Superconductivity Conference,

Chicago, Illinois, August 23-28, 1992 


\section{Disclaimer}

This report was prepared as an account of work sponsored by an agency of the United States Government. Neither the United States Government nor any agency thereof, nor any of their employees, makes any warranty, express or implied, or assumes any legal liability or responsibility for the accuracy, completeness, or usefulness of any information, apparatus, product, or process disclosed, or represents that its use would not infringe privately owned rights. Reference herein to any specific commercial product, process, or service by trade name, trademark, manufacturer, or otherwise, does not necessarily constitute or imply its endorsement, recommendation, or favoring by the United States Government or any agency thereof. The views and opinions of authors expressed herein do not necessarily state or reflect those of the United States Government or any agency thereof. 


\title{
AC Loss Measurements of Model and Full Size 50mm SSC Collider Dipole Magnets at Fermilab
}

\author{
J.P. Ozelis, S. Delchamps, S. Gourlay, T. Jaffery, W. Kinney, W. Koska, M. Kuchnir, M.J. Lamm, P.O. Mazur, D. Orris, \\ J. Strait, and M. Wake* \\ Fermi National Accelerator Laboratory \\ Batavia, Illinois, 60510
}

J. Dimarco, J. Kuzminski, and H. Zheng

SSC Laboratory, Dallas, Texas 75237

\begin{abstract}
Tests have recently been performed at Fermilab in order to measure the energy losses due to eddy currents and iron and superconductor magnetization. These measurements were performed on six $1.5 \mathrm{~m}$ long model magnets and eight $15 \mathrm{~m}$ long full scale collider dipole magnets. AC losses were measured as a function of ramp rate using sawtooth ramps from 500 to 5000 Amps for both types of magnets, while bipolar studies were additionally performed on some of the short magnets. The measured magnet voltage and current for a complete cycle are digitally integrated to yield the energy loss per cycle. Measurement reproducibility is typically $\mathbf{5 \%}$, with good agreement between long magnet measurements and extrapolations from short magnet measurement results. Magnetization loss measurements among similar magnet types agree to within experimental error, while eddy current losses correlate strongly with the observed dependence of quench current on ramp rate.
\end{abstract}

\section{INTRODUCTION}

Superconducting magnets do not ordinarily dissipate energy when energized with a constant current. However, when operated in a non-constant current mode, energy is dissipated in the form of heat due to eddy currents generated in the composite copper-niobium titanium conductor, as a consequence of coupling of the superconducting filaments in a given strand, and coupling between strands in a cable through contact resistance. Eddy currents are also generated in other conductive components of the magnet, but these provide a minor contribution. There are additional energy losses due to the motion of fluxoids throughout the superconductor in response to a changing external magnetic field, known as superconductor magnetization losses, and energy losses due to iron magnetization resulting from magnetic domain movement and re-structuring in the ferromagnetic components of the magnet. These various mechanisms lead to a net energy loss of the system when a superconducting magnet is operated in an $\mathrm{AC}$ fashion, hence they are known as $\mathrm{AC}$ losses.

The energy dissipated through these mechanisms manifests itself as a temperature rise in the conductor of the magnet. This temperature rise, if large enough, can cause the magnet to undergo a spontaneous quench. This has been observed in ramp rate studies of full size and model SSC Collider dipoles [1,2].It is therefore important to understand

Work supported by U.S. Department of Energy

Manuscript received 24 August 1992

* Present Address : SSC Laboratory, Dallas, Texas and measure this behavior, in order to define operational limits for superconducting magnets under AC operation. While the dipole magnets for the SSC collider will not be subject to fast ramp conditions, and therefore, will not be sensitive to conductor heating from $A C$ loss energy dissipation, the magnets of the High Energy Booster will be energized/de-energized at higher rates, and will consequently be more strongly affected by AC loss mechanisms. Since the HEB and collider dipoles are similar in design, measurements of $\mathrm{AC}$ losses in collider dipoles yield information useful in the design considerations of the HEB magnets. Furthermore, measurements of $\mathrm{AC}$ losses in collider dipoles provide a means for correlating eddy current losses to observed degradation of quench current as a function of ramp rate, and may also be useful in understanding ramp-related distortions of field harmonics [3].

\section{MEASUREMENT TECHNIQUES}

\section{A. Previous Methods}

Previous techniques for measuring AC losses of superconducting magnets employed either calorimetric methods or electrical methods, Calorimetric methods typically employ the vaporization of liquid helium as the mechanism by which the energy dissipation is measured [4]. This method suffers the disadvantage of a long system time constant and the necessity of carefully controlling heat leaks external to the magnet. Calorimetric methods using temperature controlled feedback of a superfluid helium bath have, however, increased the accuracy of this technique [5].

Energy losses in superconducting magnets can be observed electrically as the difference between the energy injected into and extracted from the magnet during a ramp cycle. The integration of the product of magnet voltage and current over a ramp cycle represents this energy difference. Electrical methods have historically used the technique of analog or digital integration in conjunction with a "bucking coil", whereby the magnet voltage was integrated over time and the area of the integrated voltage versus current curve taken as the energy loss $[6,7,8]$. These methods required careful adjustment of the bucking coil voltage, in order to accurately subtract the inductive component of the magnet voltage and maintain measurement precision.

\section{B. Present Method}

The present measurement technique is completely digital in nature, utilizing two HP Model 3457A digital integrating 
voltmeters to simultaneously measure magnet voltage and current. One voltmeter measures magnet voltage directly via a set of voltage taps on the superconducting coil. The other voltmeter measures the magnet current in conjunction with a $12 \mathrm{kA}$ shunt for the short magnets, and a Holec transductor for the long magnets. As the total energy flow into and out of the magnet is quite large compared to its stored energy, slight relative timing errors between the magnet voltage and current signal can lead to large errors in the loss measurement. This is avoided by simultaneously externally triggering the DVM's using a Wavetek Model 75 function generator, which provides a burst of square waves as trigger signals. The components of the measurement system, shown in Figure 1, are controlled and data is acquired by a PC via the GPIB bus. The data acquisition program is written using the ASYST programming environment.

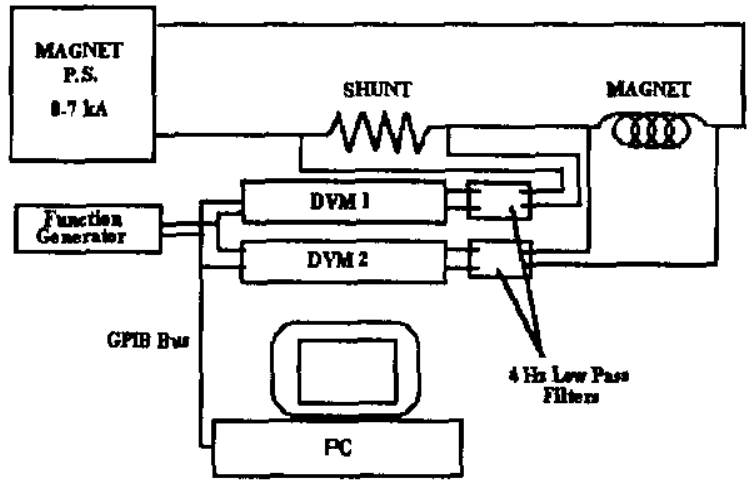

Fig. 1 The AC Loss Measurement System

In order to reduce the effects of line-cycle noise on the measurement, the voltmeters are configured to integrate the input reading over 10 power line cycles $(0.167$ secs.) Individual readings are stored in internal memory of the DVM's, to be read out in FIFO mode by the PC. The DVM's are triggered at a frequency of $4.5 \mathrm{~Hz}(0.222$ seconds). The DVM's have a dead time of $0.056 \mathrm{sec}$, , corresponding to the discharge time of an internal integrating capacitor, and other instrument-specific overhead.

The incidence of magnetization change in the superconductor, which is the primary contribution to magnetization losses, occurs at the edges of the current ramp, where the ramp rate is not a constant. This typically occurs over a 0.100 second interval, which is shorter than the measurement period of 0.222 seconds. To improve the measurement accuracy during these sections of the ramp cycle, low pass filters with a time constant on the order of the DVM dead time can be used on the voltmeter inputs. These filters also eliminate noise resulting from the SCR firing pattern of the magnet power supplies. Low pass filters of cutoff frequency $4.0 \mathrm{~Hz}$ (time constant 0.040 seconds) are therefore used on the magnet voltage and current DVM inputs. The time constants of these filters were carefully matched to preclude any systematic error in the resultant measurements. Additionally, the DVM's are operated at identical range settings, to ensure that their input impedances are identical. Further improvements in signal-to-noise ratio were realized through careful shielding and isolation of signal cables. Shielded co-axial cable is used throughout for magnet voltage measurements, while shielded twisted pair conductor is used for the shunt voltage measurements.

\section{ANALYSIS}

The product of magnet voltage $(V)$ and current $(I)$ is integrated numerically using a finite number of data points. In practice, careful consideration must be made regarding the completeness of the integration cycle, and sources of numerical errors. If the endpoints of the integration cycle are erroneously chosen such that the current at those points is not equal, then the difference in stored magnet energy at those points will be mistakenly added to the calculated loss. In the case of a full length SSC collider dipole, a difference in the starting and ending currents of $1 \mathrm{~A}$ leads to 75 Joules of stored energy being counted as a contribution to the energy loss.

A good completeness check for a cycle is provided by the following :

$$
\oint V d t=0
$$

The integration endpoints are chosen so as to best achieve this condition. To reduce the inaccuracy due to incompleteness of the measurement cycle, it is desirable to subtract the inductive component of the magnet voltage before integration, so that the subtraction of two large, roughly equal numbers, and concomitant numerical errors, can be avoided. The energy loss $U$ is calculated using

$$
\begin{aligned}
U & =\oint I(t) V(t) d t=I(0) \oint V(t) d t-\oint \int_{0}^{t} V\left(t^{\prime}\right) d t^{\prime} \frac{d I}{d t^{\prime}} d t^{\prime} \\
& =-\oint\left[\int_{0}^{t} V\left(t^{\prime}\right) d t^{\prime}-L I\left(t^{\prime}\right)+L I(0)\right] d I
\end{aligned}
$$

where $L$ is the inductance of the magnet, which changes with current due to iron saturation. For a full length SSC collider dipole magnet, we have parameterized the current dependence of the inductance as:

$L=L_{0}\left\{1-0.06(1 / 5000)^{?}\right\}$

This integration technique differs from previous methods in that it eliminates the need for a bucking coil, and uses a nonconstant magnet inductance in the subtraction of the inductive component of the magnet voltage.

\section{RESULTS}

\section{A. Short Magnets}

Measurements of AC losses were performed as a function of ramp rate for six 1.5 meter model SSC collider dipole magnets at the Fermilab Advanced Magnet R\&D facility [9]. The ramp cycle for these measurements was essentially a trapezoidal ramp from 500 to 5000 to $500 \mathrm{Amps}$, with 5 second dwells at the maximum and minimum currents. The 
ramp rates (I-dot) studied ranged from 30 to $300 \mathrm{~A} / \mathrm{sec}$. Measurements were taken on the 4th and subsequent cycles of a series of (typically) 10 ramp cycles. The results are listed in Table 1, and plotted in Figure 2. The reproducibility of these measurements is about $3 \%$ at present. We find the loss per cycle is essentially linearly dependent upon ramp rate, with a slope ranging from 0.53 to $0.76 \mathrm{~J} / \mathrm{A} / \mathrm{sec}$. This slope, representing losses due to eddy current, correlates with the ramp rate dependence of quench current (Iq), as seen in Figure 3.

Table 1. Short Magnet AC Loss Results

\begin{tabular}{|c|c|c|}
\hline Magnet & $\begin{array}{c}\text { Eddy Current } \\
\text { Loss (J/A/sec) }\end{array}$ & $\begin{array}{c}\text { Hysteresis Loss } \\
(\mathrm{J})\end{array}$ \\
\hline DSA323 & 0.53 & 99 \\
\hline DSA324 & 0.76 & 100 \\
\hline DSA328 & 0.61 & 101 \\
\hline DSA329 & 0.56 & 102 \\
\hline DSA331 & 0.62 & 97 \\
\hline DSA332 & 0.70 & 100 \\
\hline
\end{tabular}

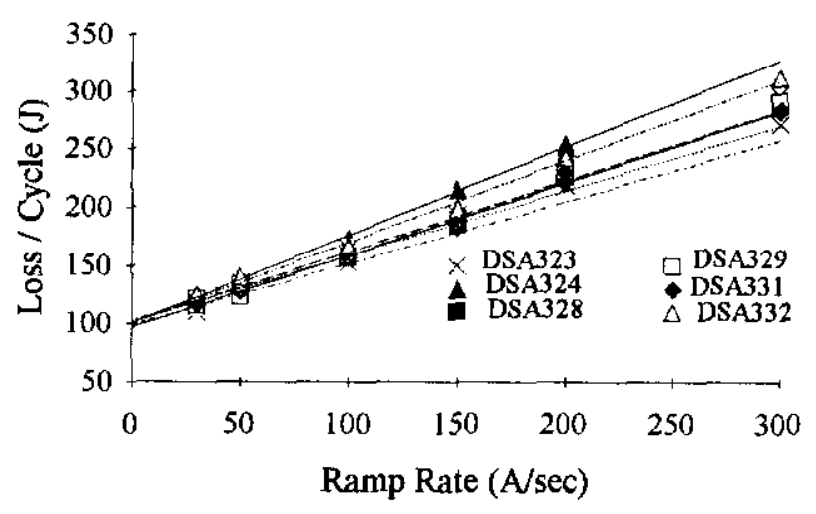

Figure 2. Short Magnet AC Loss

The intercept of the linear fit to the ramp rate data corresponds to the AC loss due to superconductor hysteresis and iron domain motion, and varies from 97 to 102 Joules, with an average value of $99.8 \pm 1.6$ Joules $(1.6 \%)$. This loss depends primarily upon characteristics of the superconducting cable, maximum field change, and magnet cross-section design. It is therefore reasonable to expect that magnets of similar design exhibit similar hysteresis losses. This behavior is evident (to within experimental error) in the hysteresis loss results for the set of short magnets tested

Bipolar studies [10] were also performed on two of the short magnets (DSA324 and DSA328). The bipolar ramps were essentially sawtooth ramps from 0 to $\pm 5000 \mathrm{Amps}$, with 5 second dwells at minimum, maximum, and zero current. The results of these measurements can be found in Table 2. In general we find higher hysteresis losses under bipolar operation, owing to the greater effects of iron and superconductor magnetization when the field changes sign. The eddy current losses increase by about a factor of 2 , as expected.

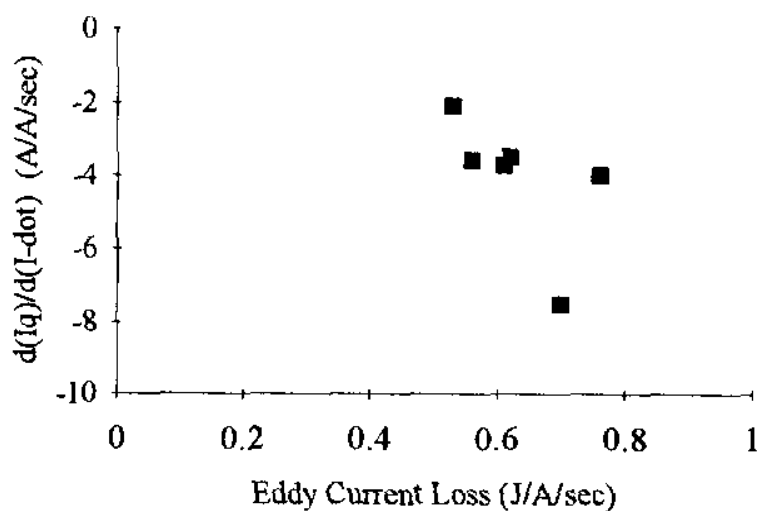

Figure 3. Eddy Current Loss vs. Ramp Rate Dependence of Quench Current - Short Magnets .

Table 2. Short Magnet AC Loss Results - Bipolar

\begin{tabular}{|c|c|c|}
\hline Magnet & $\begin{array}{c}\text { Eddy Current } \\
\text { Loss }(\mathrm{J} / \mathrm{A} / \mathrm{sec})\end{array}$ & $\begin{array}{c}\text { Hysteresis Loss } \\
(\mathrm{J})\end{array}$ \\
\hline DSA324 & 1.69 & 300 \\
\hline DSA328 & 1.41 & 309 \\
\hline
\end{tabular}

\section{B. Long Magnets}

Eight long (15 meter) magnets were tested in a similar fashion at the Fermilab Magnet Test Facility [11]. AC losses of these magnets were measured as a function of ramp rate for ramp rates of 30 to $125 \mathrm{~A} / \mathrm{sec}$. (Difficulties in ramping down the long magnets at ramp rates greater than $125 \mathrm{~A} / \mathrm{sec}$ precluded performing AC loss measurements on long magnets at high ramp rates.) The results are listed in Table 3 and plotted in Figure 4. The average hysteresis loss for this set of magnets is $\mathbf{7 4 8}$ Joules, with a standard deviation of $\mathbf{2 5}$ Joules $(3.3 \%)$.

Table 3. Long Magnet AC Loss Results Cable Type : $\mathrm{S}=$ Suprecon, $\mathrm{I}=\mathrm{IGC}, \mathrm{O}=\mathrm{Oxford}, \mathrm{K}=$ Outokumpu

\begin{tabular}{|c|c|c|c|}
\hline Magnet & $\begin{array}{c}\text { Eddy Current } \\
\text { Loss }(\mathrm{J} / \mathrm{A} / \mathrm{sec})\end{array}$ & $\begin{array}{c}\text { Hysteresis } \\
\text { Loss }(\mathrm{J})\end{array}$ & $\begin{array}{c}\text { Cable Type } \\
\text { Inner/Outer }\end{array}$ \\
\hline DCA311 & 12.0 & 744 & $\mathrm{~S} / \mathrm{I}$ \\
\hline DCA312 & 63.0 & 739 & $\mathrm{I} / \mathrm{I}$ \\
\hline DCA314 & 35.7 & 759 & $\mathrm{I} / \mathrm{I}$ \\
\hline DCA315 & 49.7 & 769 & $\mathrm{I} / \mathrm{I}$ \\
\hline DCA318 & 7.5 & 723 & $\mathrm{O} / \mathrm{K}$ \\
\hline DCA319 & 9.4 & 713 & $\mathrm{O} / \mathrm{O}$ \\
\hline DCA320 & 11.2 & 738 & $\mathrm{I} / \mathrm{S}$ \\
\hline DCA321 & 10.0 & 795 & $\mathrm{O} / \mathrm{S}$ \\
\hline
\end{tabular}

The eddy current losses, as determined by the slope of the loss/cycle vs. ramp rate data for ramp rates $\leq 75 \mathrm{~A} / \mathrm{sec}$., show large variations among magnets, ranging from 7.46 to 63.0 $\mathrm{J} / \mathrm{A} / \mathrm{sec}$. However, similar eddy current losses are observed in magnets made with cable from the same vendor. Variations in eddy current losses between magnets are most likely due to differences in the strand to strand coupling of the cable, 
which depends upon the contact resistance of the strands, and may arise from different surface treatments of the conductor, as by-products of the various cable manufacturing processes. Magnets with low eddy current losses also seemed to exhibit a non-linear ramp rate dependence at higher ramp rates. This non-linearity is not evident, however, in magnets where eddy current losses were large. It has been suggested that this nonlinearity may be due to the non-linear $V-I$ characteristics of semiconducting copper oxide, or an apparent magnetization due to trapped persistent currents [12].

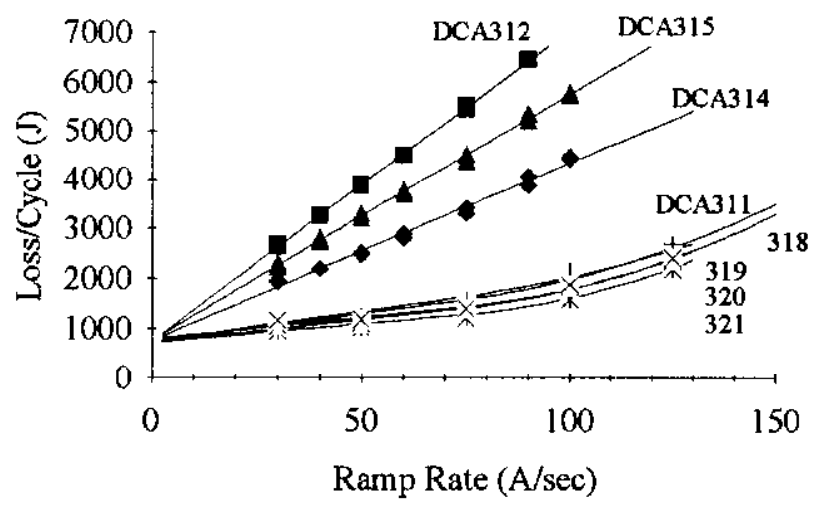

Figure 4. Long Magnet AC Loss

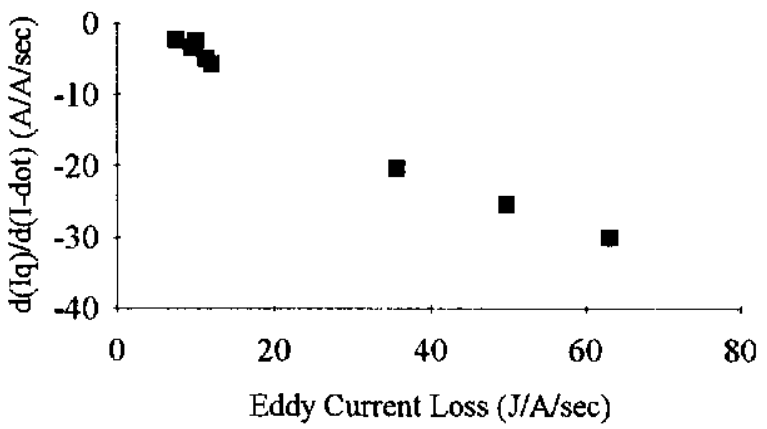

Figure 5. Eddy Current Loss vs. Ramp Rate Dependence of Quench Current - Long Magnets.

The ratio of hysteresis losses between short and long magnets is about 7.5, whlle the ratio of superconductor volume (by which the hysteresis loss should scale) is about 10. No suitable explanation for this discrepancy is presently available. The eddy current losses, however, tend to scale reasonably well with the superconductor volume ratio, at least in the case of the low eddy current loss long magnets.

In Figure 5 the relationship between eddy current losses and the degradation of quench current (Iq) (as parameterized by the slope of Iq vs. I-dot data for I-dot $\geq 75 \mathrm{~A} / \mathrm{sec}$ ) with ramp rate is plotted. These data suggest that eddy current heating in the superconducting cable is a primary mechanism for reduced quench currents at high ramp rates.

\section{CONCLUSION}

Measurements of AC losses in full size and model SSC collider dipoles have been performed using a completely digital technique. The measurement reproducibility is found to be better than $5 \%$ (typically about $3 \%$ ), and good correlation exists between measured eddy current losses and observed quench current degradation at high ramp rates. We further find excellent consistency among hysteresis losses for similar magnet designs.

\section{ACKNOWLEDGMENTS}

The authors would like to thank the technical staffs of the Fermilab Magnet Test Facility (MTF) and Advanced Magnet R\&D Facility (Lab 2) for their assistance.

\section{REFERENCES}

[1] J. Strait,et. al., "Quench Performance of Fermilab/General Dynamics Built Full Length SSC Collider Dipole Magnets", in "Proceedings of the 4th IISSC", New Orleans, LA., March 1992.

[2] T. Jaffery, et. al., "Test Results of Post ASST Design Fermilab Built 1.5 Meter SSC Dipole Magnets", paper LOA6 , this conference.

[3] Y. Zhao, et. al., "Current Dependence of Harmonic Field Coefficients of $5 \mathrm{~cm}$ Aperture $15 \mathrm{~m}$ Long SSC Dipole Magnet Prototypes", paper LOA-8, this conference.

[4] M. N. Wilson, Superconducting Magnets, New York: Oxford University Press, 1983.

[5] P. Chaumette, et. al., "A Large 1.8K Facility for Magnet Test":, Adv. in Cry. Eng., vol. 35, 269 (1990).

[6] M. N. Wilson, "An Improved Technique for Measuring Hysteresis Loss in Superconducting Magnets", Cryogenics, vol. 13, 361 (1973).

[7] A.D. McInturff, et. al., "Magnetization Characterization Study for the Energy Saver/Doubler Dipoles", IEEE Trans. Nucl. Sci., vol 28, 3211 (1981).

[8] M. Wake, et. al.,"AC Loss in Energy Doubler Magnets", IEEE Trans. Mag. vol. 15, 137 (1979).

[9] M. J. Lamm, et. al., "A Facility to Test Short Superconducting Accelerator Magnets at Fermilab", paper LOD-6, this conference.

[10] M. J. Lamm, et. al., "Bipolar and Unipolar Tests of $1.5 \mathrm{~m}$ Model SSC Collider Dipole Magnets at Fermilab", in Conference Record of the 1991 IEEE Particle Accelerator Conference, San Francisco, CA., May 1991.

[11] J. Strait, et. al., "Fermilab R\&D Test Facility for SSC Magnets", in Supercollider I, M. McAshan, ed., Plenum Press, New York, 1989.

[12] W. Wake, et. al., "AC Loss Measurements of SSC Dipole Magnets", presented at the ICFA Workshop, KEK, Japan, June 1992. 